\section{Hearing loss is an under- recognized complication of diabetes mellitus}

Limited clinical and epidemiologic evidence suggests that diabetes mellitus might be associated with an increased prevalence of hearing loss. Bainbridge and colleagues have now tested this hypothesis by evaluating data derived from the National Health and Nutrition Examination Survey (NHANES), a US-based national survey of noninstitutionalized adults.

The authors evaluated 5,140 individuals aged 20-69 years who had undergone audiometric testing. Hearing was assessed over two frequency ranges (low/mid and high) and the severity of hearing loss defined as mild or greater (pure tone average hearing threshold $>25$ decibels) or moderate or greater ( $>40$ decibels). Diagnosis of diabetes mellitus was made on the basis of selfreport. The prevalence of low/mid frequency hearing loss of mild or greater severity was higher among individuals with diabetes mellitus than those without diabetes mellitus $(21.3 \%$ vs 9.4\%). A similar result was observed for highfrequency hearing loss of mild or greater severity $(54.1 \%$ vs $32.0 \%)$. Hearing loss was also detected in individuals with impaired fasting glucose. The association of diabetes mellitus with hearing loss was found to be independent of other known risk factors (e.g. noise exposure). Limitations included self-report of diabetes mellitus and the inability to distinguish etiology of diabetes mellitus.

The findings of this study suggest that hearing loss could represent an under-recognized complication of diabetes mellitus. Routine audiometric testing might, therefore, be considered in this patient group.

Original article Bainbridge KE et al. (2008) Diabetes and hearing impairment in the United States: audiometric evidence from the National Health and Nutrition Examination Survey, 1999 to 2004. Ann Intern Med 149: 1-10

\section{Variable suppression of TSH by human chorionic gonadotropin during early pregnancy}

An inverse relationship has been described between maternal serum TSH and human chorionic gonadotropin (hCG) levels during early pregnancy. TSH levels reach a nadir at around 10 weeks' gestation, at which time hCG levels peak. In addition, free $\mathrm{T}_{4}$ levels might be elevated, although other features of hyperthyroidism are usually absent.

Haddow and colleagues evaluated the associations between TSH, free $\mathrm{T}_{4}$ and hCG levels at 11-18 weeks' gestation in a cohort of 9,562 pregnant women enrolled in the multicenter FaSTER (First and Second Trimester Evaluation of Risk for Aneuploidy) trial. Biochemical analysis was performed on paired first and second trimester serum samples collected between 1999 and 2002. Strong associations $(P \leq 0.0005)$ were detected in both trimesters between maternal covariates and serum hormone levels. These associations included BMI (hCG and free $\mathrm{T}_{4}$ ), tobacco smoking (hCG, TSH and free $\mathrm{T}_{4}$ ), gravidity (hCG, TSH and free $\mathrm{T}_{4}$ ), ethnic background (hCG and TSH), and maternal age (free $\mathrm{T}_{4}$ ). The correlations between TSH and hCG levels and between hCG and free $\mathrm{T}_{4}$ levels were weak in both trimesters. Of note, however, the TSH percentile seemed to determine susceptibility to suppression by hCG, as TSH concentrations in the lower percentiles became sharply and selectively lower at higher hCG levels.

The authors speculate that hCG might be the primary factor responsible for stimulation of the thyroid gland during early pregnancy.

Original article Haddow JE et al. (2008) Variability in thyroid stimulating hormone suppression by human chorionic gonadotropin during early pregnancy. J Clin Endocrinol Metab 93: 3341-3347

\section{Dementia in elderly people with diabetes is predicted by microalbuminuria}

Previous studies suggested that microalbuminuria might contribute to the risk of diabetes-related dementia in elderly people. In a longitudinal cohort study, Bruce and colleagues investigated the role of microalbuminuria in clinically relevant cognitive decline in non-demented elderly people with type 2 diabetes mellitus.

All participants ( $n=205$, mean age 75.3 years) underwent clinical assessment and an initial cognitive screening followed by a detailed assessment of cognitive status, which was repeated a median 1.6 years later. At initial assessment, 164 patients had normal cognition and 41 had mild cognitive impairment without dementia. At follow-up, 33 participants had cognitive decline, including 4 new cases of dementia and 29 new cases of cognitive impairment without dementia. 